\title{
A snapshot full-Stokes spectropolarimeter for detecting life on Earth
}

\author{
Frans Snik ${ }^{\mathrm{a}}$, Christoph U. Keller ${ }^{\mathrm{a}}$, David S. Doelman ${ }^{\mathrm{a}}$, Jonas Kühn ${ }^{\mathrm{b}}$, C. H. Lucas Patty ${ }^{\mathrm{c}}$, \\ H. Jens Hoeijmakers ${ }^{\mathrm{b}, \mathrm{d}}$, Vidhya Pallichadath ${ }^{\mathrm{e}}$, Daphne M. Stam ${ }^{\mathrm{e}}$, Antoine Pommerol ${ }^{\mathrm{b}}$, Olivier \\ Poch $^{\mathrm{f}}$, and Brice-Olivier Demory ${ }^{\mathrm{b}}$ \\ aLeiden Observatory, Leiden University, P.O. Box 9513, 2300 RA Leiden, The Netherlands \\ ${ }^{b}$ Physikalisches Institut, Universität Bern, Sidlerstrasse 5, 3012, Bern, Switzerland \\ ${ }^{c}$ Biological Research Centre, Hungarian Academy of Sciences, P.O. Box 521, H-6701 Szeged, \\ Hungary \\ dObservatoire de Genève, Université de Genève, Chemin des Maillettes 51, 1290 Versoix, \\ Switzerland \\ eFaculty of Aerospace Engineering, Delft University of Technology, Kluyverweg 1, 2629 HS \\ Delft, The Netherlands \\ ${ }^{f}$ Univ. Grenoble Alpes, CNRS, IPAG, 38000 Grenoble, France
}

\begin{abstract}
We present the design of a point-and-shoot non-imaging full-Stokes spectropolarimeter dedicated to detecting life on Earth from an orbiting platform like the ISS. We specifically aim to map circular polarization in the spectral features of chorophyll and other biopigments for our planet as a whole. These non-zero circular polarization signatures are caused by homochirality of the molecular and supramolecular configurations of organic matter, and are considered the most unambiguous biomarker. To achieve a fully solid-state snapshot design, we implement a novel spatial modulation that completely separates the circular and linear polarization channels. The polarization modulator consists of a patterned liquid-crystal quarter-wave plate inside the spectrograph slit, which also constitutes the first optical element of the instrument. This configuration eliminates cross-talk between linear and circular polarization, which is crucial because linear polarization signals are generally much stronger than the circular polarization signals. This leads to a quite unorthodox optical concept for the spectrograph, in which the object and the pupil are switched. We discuss the general design requirements and trade-offs of LSDpol (Life Signature Detection polarimeter), a prototype instrument that is currently under development.
\end{abstract}

Keywords: Polarimetry, Biomarkers, Space Instrumentation

\section{INTRODUCTION}

Discovering extraterrestrial life is one of the greatest and most exciting scientific challenges for humanity in the 21st century. The exploration of our Solar System has led to the identification of potential habitable environments which might host life locally, in the sub-surfaces of Mars or in the liquid water reservoirs of some of the icy moons of the giant planets (Europa, Enceladus). Moreover, we are very much in the middle of the exoplanet revolution, with almost daily detections of planets orbiting other stars than the Sun. Over the last decades, the mostly indirect exoplanet detection techniques that involve measurements of starlight (e.g., radial velocity and transit methods) have unveiled the spectacular ubiquity and diversity of exoplanetary systems. It is now clear that rocky planets in the habitable zones of stars (solar-type down to M-dwarfs) are indeed very common. The next generation of telescopes, both on the ground and in space, will be optimized and equipped with advanced instrumentation for the direct observation and characterization of such potentially life-bearing planets. Through spectroscopy and polarimetry, evidence for habitability and even habitation can be accrued: ${ }^{1}$ Liquid water can be identified, for example, through the glint of starlight off a surface ocean, ${ }^{2}$ or through the occurrence of rainbows

Further author information: Frans Snik: E-mail: snik@strw.leidenuniv.nl 
that are produced by liquid-water clouds. We also need to find indications of the planetary atmosphere being out of chemical equilibrium, e.g. large amounts of molecular oxygen, which on Earth is a strong biomarker, as virtually all this oxygen is produced by photosynthesis. However, also abiotic processes, such as UV photolysis from stellar flares, can produce $\mathrm{O}_{2}$, and therefore a wide range of biomarker gases and atmospheric properties (in particular clouds) need to be detected and characterized before a detection of life can be robustly claimed.

One property of life is considered to be a universal and unambiguous biomarker: the homochirality of molecules that constitute the building blocks of life (e.g. amino acids, sugars). ${ }^{3,4}$ The occurrence of a predominance of single handedness of chiral molecules is a clear sign of biological production, as pure chemistry would produce a racemic mixture of both left- and right-handed versions. Multiple scattering of UV light in the early solar system is hypothesized to contribute to an initial enantiomeric excess that led to life on Earth picking left-handed amino acids and right-handed sugars. ${ }^{5}$ The symmetry-breaking in chiral handedness leaves a distinct imprint on starlight that is reflected/scattered off organisms built up from homochiral molecules: it becomes partially circularly polarized. Because the direction of circular polarization is determined by the chiral molecules, it is not averaged out when this reflected/scattered light signal is integrated across the planetary disk. This averaging out does occur for the circular polarization signal of starlight that is scattered by cloud and/or aerosol particles. ${ }^{6}$ Furthermore, this circular polarization generally has a specific spectral signature with both negative and positive circular polarization peaks, which makes it readily distinguishable from multiple-scattering effects that can create spectrally flat non-zero circular polarization. ${ }^{6}$

On Earth, particularly photosynthetic organisms exhibit a non-zero circular polarization spectrum, due to the homochirality of chlorophyll and other biopigments, see Ref. ${ }^{7}$ and references therein. Sparks et al. ${ }^{8,9}$ describe laboratory experiments using a scanning monochromator with a photoelastic modulator (PEM) system to measure the circular polarization spectra due to transmission and reflection of various types of leaves and microbes, and show that the signals are weak $(\sim 1-0.01 \%)$ but very pronounced. Sterzik \& Bagnulo, used a spectropolarimeter on a telescope to measure the spectral circular polarization signature of a Philondendron leaf. ${ }^{10}$ In recent years, Patty et al. ${ }^{11-14}$ have developed and utilized the TreePol instrument to enable sensitive circular spectropolarimetric measurements both in the lab and in the field. TreePol is spectrally multiplexed with fast, dual-beam ferro-electric liquid-crystal (FLC) modulation. They demonstrated in the lab that the typical antisymmetric circular polarization signal in the chlorophyll absorption band for a leaf in transmission disappears as a leaf "dies", which they attribute to the desintegration of the chiral macromolecular organization of the chlorophyll molecules as opposed to the destruction of the chlorophyll itself. ${ }^{11}$ Outside the lab, they used TreePol to obtain a clear non-detection of circular polarization for a nearby field of artificial grass, while a forest at $\sim 3 \mathrm{~km}$ distance yielded a clear remote detection of life. ${ }^{14}$

We are currently building a second version of TreePol to fly on an aircraft to provide more observations of a larger and more varied range of scenes. The TreePol design, however, cannot be adopted for a space mission for reasons explained in Sect. 3. We therefore started the design of a radical optical concept that we call LSDpol, for "life signature detection polarimeter" (although it also alludes to a few crazy design choices made in the process). The goal of this work is to design and build an instrument that can detect and map circular spectropolarimetric signatures of photosynthetic life on Earth in a large field survey, in an aircraft campaign, and in a global-mapping space mission. Our final aim with LSDpol is to observe the Earth as an exoplanet, to demonstrate that the average circular polarization signal for planet Earth is strong enough to be detected when all the sunlight it reflects is compressed into a "pale blue dot".

With large ground-based telescopes, the linearly polarized signatures of atmospheric molecular oxygen and the "green bump" and "red edge" due to vegetation have been detected in Earthshine data ${ }^{15-19}$ (i.e. sunlight that has been reflected by the Earth and then by the Moon). However, the weak circular polarization signatures are very difficult to measure in this way, as the depolarization of the lunar surface is significant and unknown, and, as the linear polarization is typically orders of magnitude larger, the polarization cross-talk issues in telescopes and instruments inhibit robust detections. ${ }^{20}$ Moreover, Earthshine observations can only be done for a limited phase angle range of the Earth. Hence, a Dutch team is developing the LOUPE instrument (Lunar Observatory for Unresolved Polarimetry of Earth $)^{21,22}$ to provide highly time-resolved linear spectropolarimetry for all phase angles as seen from the Moon (i.e. the observations should cover at least roughly a month). LOUPE does not 
(yet) include the highly dedicated observation of circular polarization as it greatly complicates the instrument design.

For the LSDpol instrument, we focus on mapping and monitoring the circular polarization spectral signatures across the Earth first from a Low Earth Orbit, e.g. from the ISS or a cubesat, and then possibly from a geo-stationary orbit or indeed the Moon. Because it is compact and robust (no moving parts), our novel instrument concept could also be used to detect extraterrestrial life, e.g. on Mars ${ }^{23}$ as observed from an orbiter or lander/rover, or on icy moons like Europa and Enceladus with a mission probing the geysers of sub-surface oceans. In the end, we aim to inform the design of high-contrast imaging instrumentation ${ }^{24-26}$ on the next generations of telescopes on the ground (the Extremely Large Telescopes targeting potentially habitable rocky planets orbiting M-dwarfs) and in space (targeting Earth analogs around sun-like stars), to ensure that their discoveries of signs of life on exoplanets are as unambiguous and compelling as possible.

\section{INSTRUMENT REQUIREMENTS}

For our LSDpol development we aim for a compact instrument with no moving parts that can be installed within the new Bartolomeo* platform of the ISS. This platform offers near-nadir observations of a large fraction of the Earth with a wide range of ecosystems (e.g. rain forests, algal blooms, deserts, and ice/snow caps), in combination with ample data bandwidth. We have imposed the following requirements on LSDpol:

- Single spatial pixel (i.e. no spatial resolution) from ISS;

- Full-Stokes (i.e. Stokes parameters $I, Q, U$, and $V$ );

- Spectral range 350-900 nm;

- Spectral resolution < $10 \mathrm{~nm}$;

- Systematic error in circular polarization $<10^{-4}$ of the intensity.

- Cross-talk from linear polarization into circular $<10^{-3}$;

- Able to integrate over many observations to approximate polarized spectrum of exoplanet Earth;

- No moving parts;

- Insensitive to temperature (given a reasonable operational range);

- Volume: 1 liter;

- Weight: 1 kg;

- Power: $\sim 1 \mathrm{~W}$.

Before a full science mission on the ISS, we aim to deploy a prototype in the field and on an aircraft. Moreover, the design should also be compatible with a cubesat mission (with more limited data bandwidth).

\section{POLARIZATION MODULATION APPROACH}

The greatest challenge for LSDpol are the requirements on the polarimetric performance, ${ }^{27,28}$ and those thus drive its entire design. The TreePol instrument combines several polarization modulation techniques to ensure high sensitivity for circular polarization whilst being insensitive to linear polarization signals, which are usually several orders of magnitude larger. ${ }^{11}$ TreePol features a Fresnel rhomb that turns circular polarization into linear polarization at $\pm 45^{\circ}$, which is consecutively measured by a rapidly switching FLC that is synchronized with the spectrograph detectors. The polarization analyzer is a polarizing (wire-grid) beam-splitter that feeds two fiber-based spectrometers that are also synced to each other. In this way, the two circular polarization states

\footnotetext{
${ }^{*}$ https://directory.eoportal.org/web/eoportal/satellite-missions/i/iss-bartolomeo
} 
are modulated in time as rapidly as possible to minimize the influence of time-variable effects that could mimic actual variation in polarization signals (e.g. swaying trees). The dual-beam implementation adds another layer of mitigation of temporal systematics, and also cancels the differential effects between the two spectrograph channels to first order. ${ }^{29}$ In addition, a rotating half-wave plate in front of the instrument effectively depolarizes linear polarization while it merely flips the sign of circular polarization. This eliminates linear $\rightarrow$ circular polarization cross-talk particularly by non-ideal effects of the FLC (e.g. imperfect switching angle, polarized spectral fringes).

Unfortunately, we consider this TreePol implementation to be generally unsuitable for space applications. First, although a different kind of liquid-crystal modulation has recently been space-qualified, ${ }^{30}$ having an actively switching liquid-crystal element as the primary polarization modulator adds to the risk of instrument failure. Moreover, in space, a fast rotating half-wave plate should be compensated by a counter-rotating element, which would further complicate the instrument. Furthermore, to assess systematic polarimetric errors as accurately as possible, we wish to also measure the linear polarization. But most importantly, we opt for a fully snapshot polarimetric implementation (thus, an instantaneous measurement of all Stokes parameters), as temporal modulation in combination with an orbiting, and possibly scanning platform could lead to spurious polarization signals.

We have therefore investigated various implementations of "channeled spectropolarimetry". ${ }^{28,31}$ The most common implementation uses two thick retarder crystals with different thicknesses (often 1:2) that yield three carrier waves that spectrally modulate $Q, U$ and $V .^{32}$ However, $U$ and $V$ are modulated on the same carriers, only with a different phase. Even a slight calibration error in the linear polarization $U$ can therefore obfuscate the much smaller circular polarization signal $V$. A dual-beam implementation is necessary to prevent aliasing between the intensity spectrum and the spectral polarization modulation carriers. ${ }^{33}$ Moreover, the spectral resolution of the polarization data product is necessarily much smaller than the intrinsic resolving power of the spectrograph. Sparks et al. ${ }^{34}$ introduced a similar implementation but with wedged birefringent prisms that provide modulation of all polarized Stokes parameters mostly in the dimension along the spectrograph slit. This ensures that the full resolution of the spectrometer can be attained, and many photons can be collected in one shot on a two-dimensional detector, which facilitates a high polarimetric sensitivity. However, $U$ and $V$ are still situated on the same carriers, which greatly enhances the risk of cross-talk problems. Moreover, the authors reformat light from a target by means of cylindrical fore-optics, which are also likely to induce cross-talk and instrumental polarization.

A new solution to full-Stokes modulation was developed independently by Sparks et al. ${ }^{35}$ and the authors of this paper. The implementation is very similar to a classical rotating wave plate polarimeter that yields a modulation cf.:

$$
I^{\prime}(\delta, \theta)=\frac{1}{2}\left(I+\left[\frac{1}{2} Q((1+\cos \delta)+(1-\cos \delta) \cos 4 \theta)+\frac{1}{2} U(1-\cos \delta) \sin 4 \theta-V \sin \delta \sin 2 \theta\right]\right) .
$$

From Eq. 1 it is clear that circular and linear polarization are modulated in different channels $(2 \theta$ and $4 \theta$, respectively) that are strictly orthogonal regardless of the calibration error, and that the modulation efficiency is maximized for Stokes $V$ when the retarder is a quarter-wave plate (i.e. when $\delta=\pi / 2$ ). Note that linear polarization is then still modulated with half the efficiency. We implement this modulation approach as a spatial modulation with a patterned quarter-wave retarder that is co-located with the spectrograph slit. The corresponding modulated spectra for $100 \% Q, U$, and $V$ are presented in Fig. 1, which demonstrates the individual channels for linear and circular polarization. We choose to implement this method in combination with a polarizing beam-splitter, that allows for the mitigation of the intensity imbalance in Stokes $Q$ (see Eq. 1 and the left and right top panels in Fig. 1), and, more importantly, issues that arise when the slit is not homogeneously illuminated and/or not uniform in width. ${ }^{29}$

The required patterned quarter-wave plate is readily produced using state-of-the-art liquid-crystal techniques. A direct-write technique ${ }^{36}$ is used to impose any fast-axis orientation pattern onto any optical substrate, with a resolution down to $\sim 1 \mu \mathrm{m}$. Next, several layers of liquid crystals with different properties (type of molecule, layer thickness, twist) can be combined to yield close-to-quarter-wave retardance over a large spectral bandwidth (e.g. the entire visible range). ${ }^{37}$ Such a quarter-wave plate resembles a so-called "polarization grating", ${ }^{38,39}$ that very effectively splits circular polarization into diffraction orders \pm 1 when the retardance is half-wave, and the 


\section{patterned QWP spatial modulation}
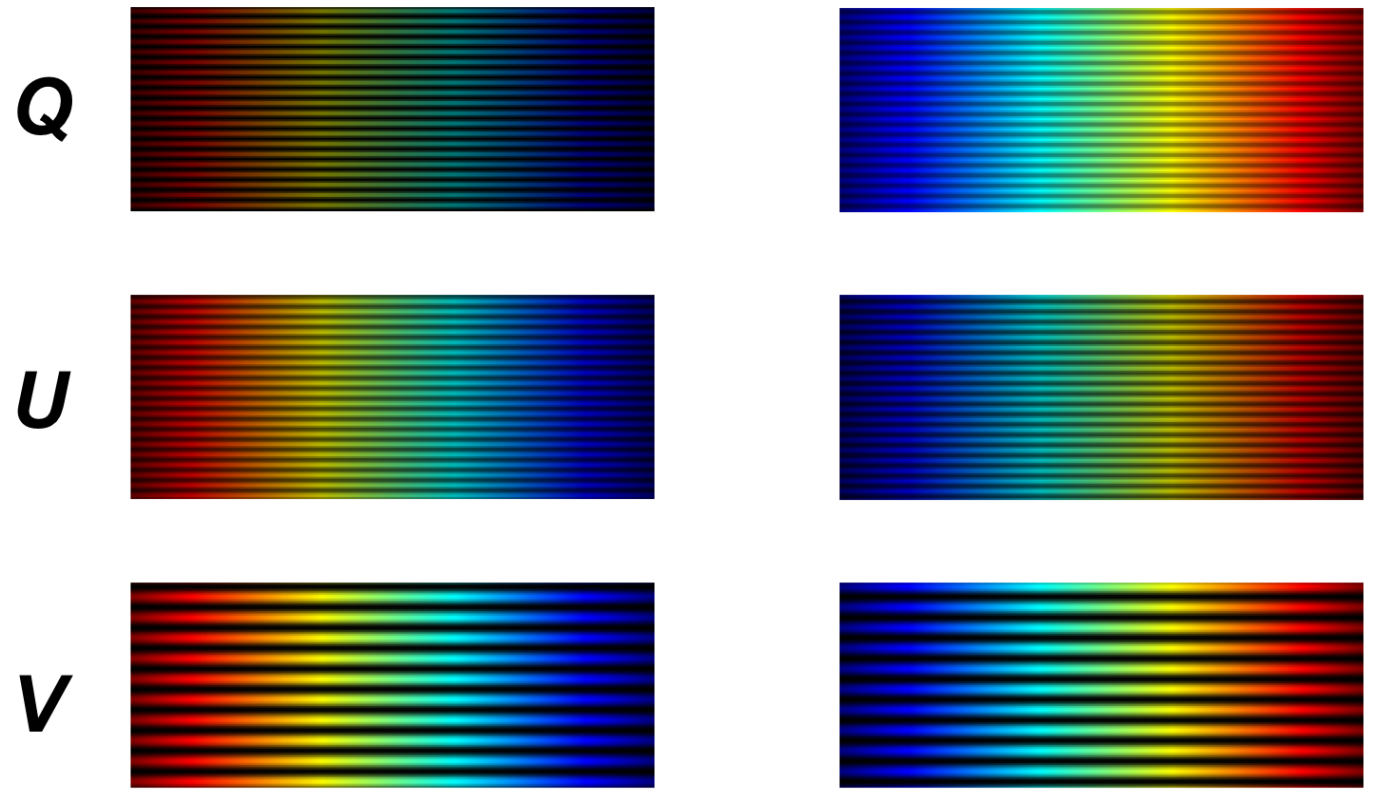

Figure 1. Dual-beam (linear polarization splitting) implementation of spatial modulation by a patterned quarter-wave retarder at the slit for $100 \% Q, U$, and $V$, see Eq. 1 .

Error in Stokes Q,U,V due to flatfield errors with 1024 pixels

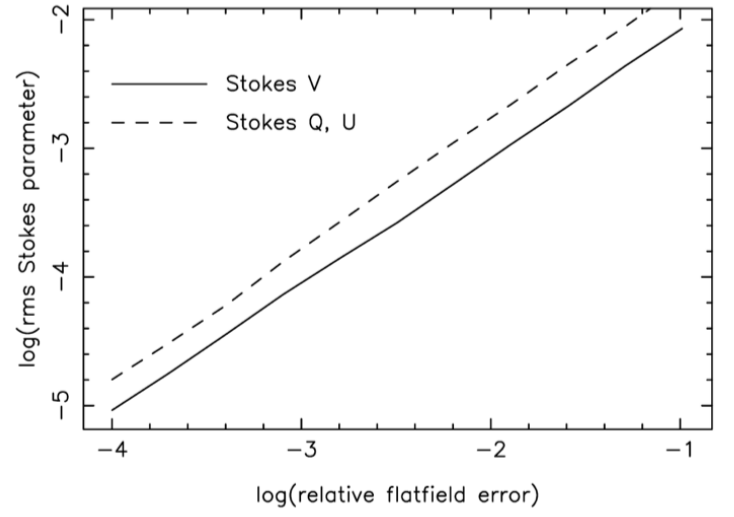

Error in Stokes $Q, U, V$ due to $1 \%$ flatfield error

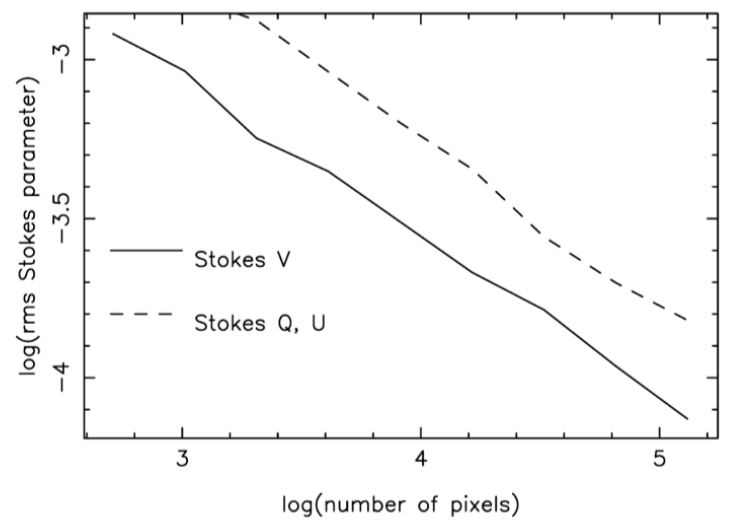

Figure 2. Simulations of spurious polarization effects due to limited flat-field calibration (pixel-to-pixel variations).

geometric phase ${ }^{40}$ dominates the optical propagation. In our case, we apply large "grating periods" and half the efficiency (because of the quarter-wave retardance) such that the diffraction effects are under control.

After the spatial modulation and the dual-beam implementation, the most important source of systematic error to the polarimetry stems from limited calibration accuracy of the pixel-to-pixel responses (the "flat-field"). Figure 2 shows how this error gets averaged down by spreading the modulation over many pixels in the crossspectral direction. A flat-field calibration accuracy of $\sim 1-0.1 \%$ is deemed feasible, which corresponds to a realistic amount of pixels $(\sim 1000)$ to achieve $\sim 10^{-4}$ polarimetric sensitivity for Stokes $V$.

To further mitigate such systematics, we experimented with an additional layer of spectral modulation. 


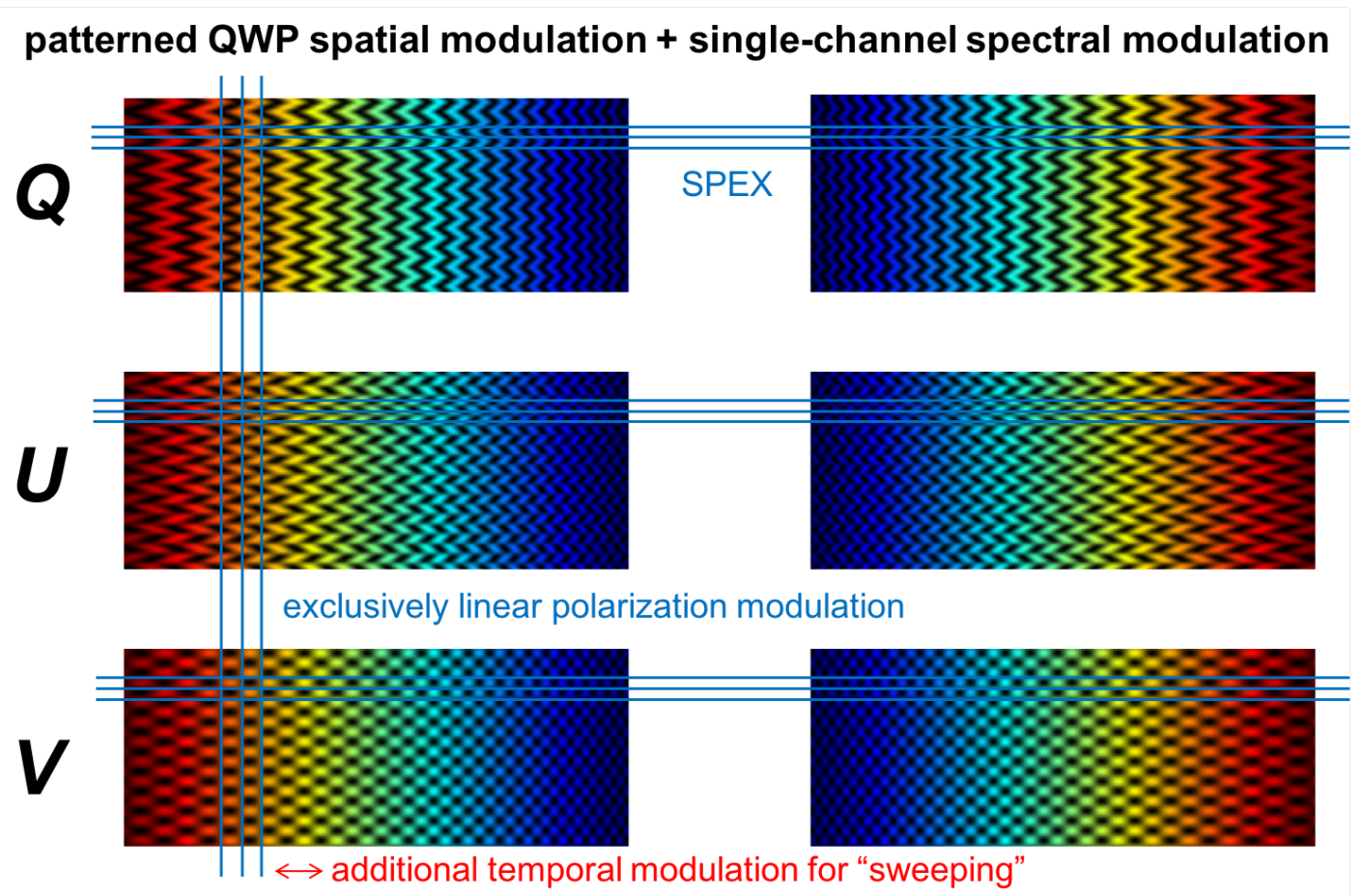

Figure 3. Modulation patterns for spatial modulation with a patterned quarter-wave retarder in combination with a single spectral modulator crystal.

patterned QWP spatial modulation + three-channel spectral modulation
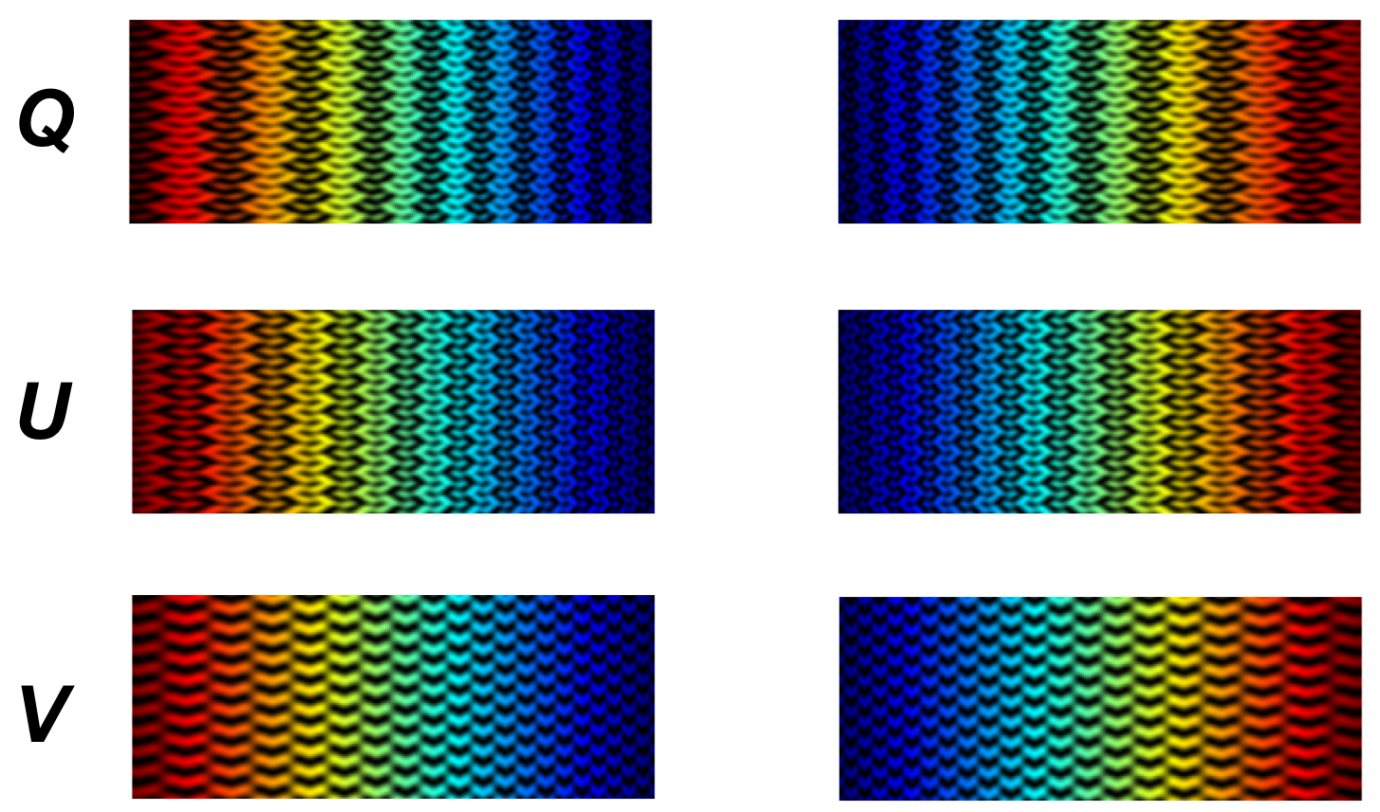

Figure 4. Modulation patterns for spatial modulation with a patterned quarter-wave retarder in combination with two spectral modulator crystals. 
Figure 3 shows the resulting modulation patterns when a single thick retarder crystal is added as a spectral polarization modulator behind the patterned quarter-wave retarder. It is clear that $Q, U$, and $V$ obtain an even more different appearance, although the modulation efficiency in linear polarization has increased. Interestingly, specific rows and columns in the modulation patterns exhibit exclusively modulation in linear polarization, that can as such be used to assess systematics. The rows in which the quarter-wave retarder is oriented at $\pm 45^{\circ}$ have a fully efficient spectral modulation for linear polarization, ${ }^{33}$ for which advanced data-analysis routines have been developed in the context of the SPEX instrument suite. ${ }^{41-43}$ Also, specific columns for which the spectral modulator has a retardance modulo $\pm \pi / 2$, there is no modulation for $V$. As linear polarization will typically be observed for a wide range of polarization angles, all these pixels can be used to assess the effects of limited flat-field calibration on the final averaged circular polarization spectrum of Earth. One more layer of temporal modulation could be added to the spectral modulator (e.g. an LCVR ${ }^{30}$ ) to sweep these pixels over the entire spectrum. In Fig. 4, we show the effect of a full-Stokes spectral modulation ${ }^{32}$ that yields even more pronounced modulation patterns. Interestingly and incidentally, Stokes $V$ appears as a pattern of little 'V's.

\section{OPTICAL DESIGN}

The adopted modulation approach drives the optical design of the spectrograph. Firstly, the spatial modulator needs to be co-located with the spectrograph slit. Secondly, the modulator and slit need to be illuminated uniformly. And thirdly, the polarization modulator should be the very first element of the optical train, to minimize the effects of cross-talk due to any fore-optics. ${ }^{44}$ This means that the slit acts both as the spectrograph slit, as well as the entrance aperture of the instrument, a veritable contradictio in terminis! To keep the spectrograph design simple, we still adopt the configuration of collimator-grating-camera, but this time the "collimator" produces an image of the target at its back focal point. This is where we locate a field stop, that, together with baffling in front of the slit, defines the field of view over which the instrument integrates for a snapshot observation. The rays for different points along the slit hit the grating under different angles, but it turns out that for the medium spectral resolution required for the simple modulation approach as shown in Fig. 1, this is acceptable. We implement an off-the-shelf polarization grating ${ }^{38,39 \dagger}$, that together with an achromatic quarterwave plate in front, combines linear polarization beam-splitting with high-efficiency broadband diffraction in spectral orders \pm 1 . A "camera" lens then reimages the slit onto the detector and produces the two complementary spectra. The ensuing optical design is sketched in Fig. 5.

As the slit determines both the spectral resolution as well as the photon flux, there is an interesting trade-off for the slit width, which for now we fix at $100 \mu \mathrm{m}$ for a tentative design for the ISS altitude and ground speed. The slit needs to be as long as possible to catch as many photons as possible, and to average out systematic effects

${ }^{\dagger}$ https://www.edmundoptics.com/f/polarization-gratings/39543/

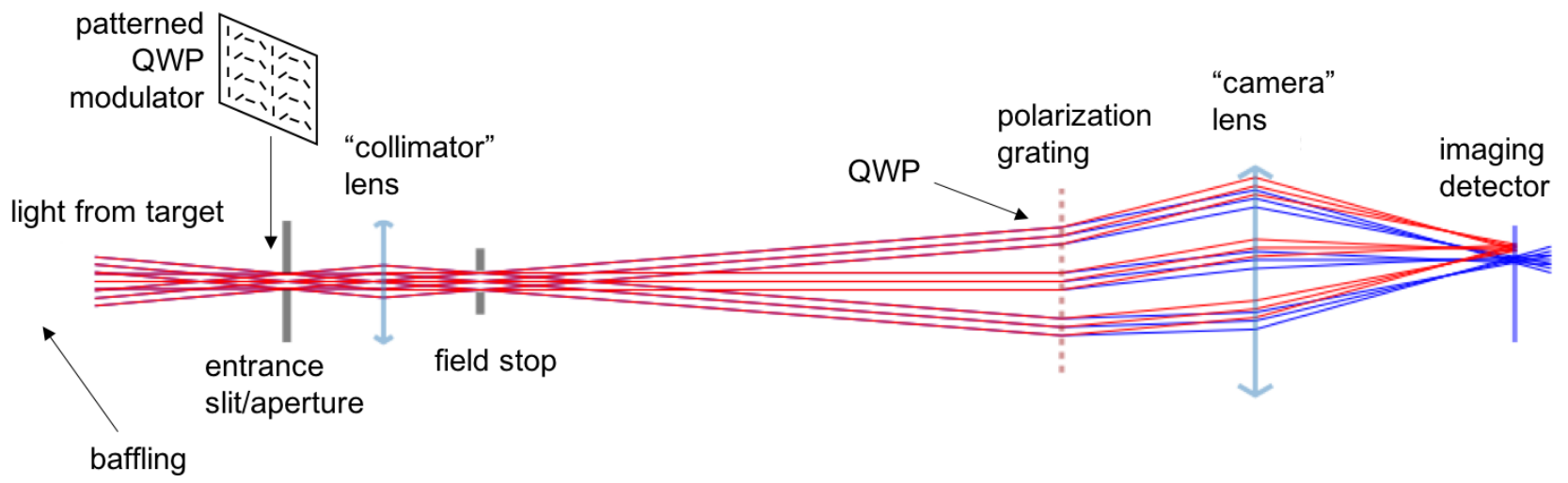

Figure 5. Sketch of the optical design for LSDpol. Only the +1 diffraction order of the polarization grating is traced. 
along the spatial polarization modulation direction. Both the slit as well as the spatial polarization modulator (acting as an inefficient polarization grating) diffract light, in perpendicular directions. In combination with the field stop, this leads to some vignetting (which also the baffling will induce). A careful analysis and a context camera will therefore be required to establish the exact footprint on Earth pertaining to the signal for every spectral recording.

\section{CONCLUSIONS \& OUTLOOK}

The challenging requirements of Sect. 2 have led us to the introduction of a novel spatial modulation approach in combination with a very unconventional spectrograph optical design (although no hallucinogens were necessary to come up with the LSDpol concept; the imagery in Figs. 1-4 was sufficient). We are currently building a prototype instrument cf. the design in Fig. 5, which will first be calibrated and tested in the lab. Next, we will deploy it in field and aircraft campaigns, and compare the results with measurements from TreePol $1 \& 2$. We will further develop and miniaturize the LSDpol instrument concept based on our experiences, and work in the context of the MERMOZ project $\ddagger$ towards an implementation for ISS/Bartolomeo (or, alternatively, a cubesat), which is the ideal platform to create a crucial map of the circular polarization signals for the only planet we currently know harbors life. This instrument is ideally flown in conjunction with other dedicated Earth-as-an-exoplanet instrumentation, such as LOUPE. Furthermore, the linear spectropolarimetry of LSDpol is ideally cross-validated with the SPEXone instrument that is being constructed for the PACE mission ${ }^{43}$ to provide essential measurements of the influence of atmospheric aerosols on our climate and our health. As such, MERMOZ/LSDpol can also contribute to Earth science, in addition to detecting life on Earth to enable the detection of life elsewhere in the universe.

\section{ACKNOWLEDGMENTS}

The research of FS and DSD leading to these results has received funding from the European Research Council under ERC Starting Grant agreement 678194 (FALCONER). This contribution of HJH has received funding from the European Research Council (ERC) under the European Unions Horizon 2020 research and innovation programme (projects Four Aces and EXOKLEIN with grant agreement numbers 724427 and 771620, respectively). JK, HJH, AP \& B-OD are acknowledging support from the NCCR PlanetS, the Swiss National Science Foundation and the University of Bern's Centre for Space and Habitability (CSH) that are funding the feasibility study of the MERMOZ project.

\section{REFERENCES}

[1] Schwieterman, E. W., Kiang, N. Y., Parenteau, M. N., Harman, C. E., DasSarma, S., Fisher, T. M., Arney, G. N., Hartnett, H. E., Reinhard, C. T., Olson, S. L., Meadows, V. S., Cockell, C. S., Walker, S. I., Grenfell, J. L., Hegde, S., Rugheimer, S., Hu, R., and Lyons, T. W., "Exoplanet Biosignatures: A Review of Remotely Detectable Signs of Life," Astrobiology 18, 663-708 (Jun 2018).

[2] Trees, V. J. H. and Stam, D. M., "Blue, white, and red ocean planets. Simulations of orbital variations in flux and polarization colors," Astronomy and Astrophysics 626, A129 (Jun 2019).

[3] Wald, G., "The Origin of Optical Activity," Annals of the New York Academy of Sciences 69, 352-368 (Aug 1957).

[4] Bonner, W. A., "Chirality and life," Origins of Life and Evolution of the Biosphere 25, 175-190 (Jun 1995).

[5] Bailey, J., Chrysostomou, A., Hough, J. H., Gledhill, T. M., McCall, A., Clark, S., Menard, F., and Tamura, M., "Circular Polarization in Star-Formation Regions: Implications for Biomolecular Homochirality," Science 281, 672 (Jul 1998).

[6] Rossi, L. and Stam, D. M., "Circular polarization signals of cloudy (exo)planets," Astronomy and Astrophysics 616, A117 (Aug 2018).

\footnotetext{
${ }^{\ddagger}$ http://www.saintex.unibe.ch/research/research_interests/
} 
[7] Patty, C. H. L., ten Kate, I. L., Sparks, William, B., and Snik, F., "Remote Sensing of Homochirality: A Proxy for the Detection of Extraterrestrial Life," in [Chiral Analysis; Advances in Spectroscopy, Chromatography and Emerging Methods, ISBN 978-0-444-64027-7], Polavarapu, P. L., ed., ch. 2, 570, Elsevier Science (2018).

[8] Sparks, W. B., Hough, J., Germer, T. A., Chen, F., DasSarma, S., DasSarma, P., Robb, F. T., Manset, N., Kolokolova, L., Reid, N., Macchetto, F. D., and Martin, W., "Detection of circular polarization in light scattered from photosynthetic microbes," Proceedings of the National Academy of Science 106, 7816-7821 (May 2009).

[9] Sparks, W. B., Hough, J. H., Kolokolova, L., Germer, T. A., Chen, F., DasSarma, S., DasSarma, P., Robb, F. T., Manset, N., Reid, I. N., Macchetto, F. D., and Martin, W., "Circular polarization in scattered light as a possible biomarker," Journal of Quantitative Spectroscopy and Radiative Transfer 110, 1771-1779 (Sep 2009).

[10] Sterzik, M. and Bagnulo, S., "Polarization of Leaves and Life," in [Astronomical Polarimetry 2008: Science from Small to Large Telescopes], Bastien, P., Manset, N., Clemens, D. P., and St-Louis, N., eds., Astronomical Society of the Pacific Conference Series 449, 399 (Nov 2011).

[11] Patty, C. H. L., Visser, L. J. J., Ariese, F., Buma, W. J., Sparks, W. B., van Spanning, R. J. M., Röling, W. F. M., and Snik, F., "Circular spectropolarimetric sensing of chiral photosystems in decaying leaves," Journal of Quantitative Spectroscopy and Radiative Transfer 189, 303-311 (Mar 2017).

[12] Patty, C. L., Luo, D. A., Snik, F., Ariese, F., Buma, W. J., ten Kate, I. L., van Spanning, R. J., Sparks, W. B., Germer, T. A., Garab, G., and Kudenov, M. W., "Imaging linear and circular polarization features in leaves with complete mueller matrix polarimetry," Biochimica et Biophysica Acta (BBA) - General Subjects 1862(6), 1350 - 1363 (2018).

[13] Patty, C. H. L., Ariese, F., Buma, W. J., ten Kate, I. L., van Spanning, R. J. M., and Snik, F., "Circular spectropolarimetric sensing of higher plant and algal chloroplast structural variations," Photosynthesis Research 140, 129-139 (May 2019).

[14] Patty, C. H. L., Loes ten Kate, I., Buma, W. J., van Spanning, R. J. M., Steinbach, G., Ariese, F., and Snik, F., "Circular spectropolarimetric sensing of vegetation in the field; possibilities for the remote detection of extraterrestrial life," Astrobiology, arXiv:1902.05859 (Feb 2019).

[15] Sterzik, M. F., Bagnulo, S., and Palle, E., "Biosignatures as revealed by spectropolarimetry of Earthshine," Nature 483, 64-66 (Mar 2012).

[16] Sterzik, M. F., Bagnulo, S., Stam, D. M., Emde, C., and Manev, M., "Spectral and temporal variability of Earth observed in polarization," Astronomy and Astrophysics 622, A41 (Feb 2019).

[17] Takahashi, J., Itoh, Y., Akitaya, H., Okazaki, A., Kawabata, K., Oasa, Y., and Isogai, M., "Phase Variation of Earthshine Polarization Spectra," Publications of the Astronomical Society of Japan 65, 38 (Apr 2013).

[18] Bazzon, A., Schmid, H. M., and Gisler, D., "Measurement of the earthshine polarization in the B, V, R, and I bands as function of phase," Astronomy and Astrophysics 556, A117 (Aug 2013).

[19] Miles-Páez, P. A., Pallé, E., and Zapatero Osorio, M. R., "Simultaneous optical and near-infrared linear spectropolarimetry of the earthshine," Astronomy and Astrophysics 562, L5 (Feb 2014).

[20] Bagnulo, S., Sterzik, M., and Fossati, L., "Characterization of the Cross Talk Between Linear and Circular Polarization for the FORS1 Instrument of the ESO VLT," in [Astronomical Polarimetry 2008: Science from Small to Large Telescopes], Bastien, P., Manset, N., Clemens, D. P., and St-Louis, N., eds., Astronomical Society of the Pacific Conference Series 449, 76 (Nov. 2011).

[21] Karalidi, T., Stam, D. M., Snik, F., Bagnulo, S., Sparks, W. B., and Keller, C. U., "Observing the Earth as an exoplanet with LOUPE, the lunar observatory for unresolved polarimetry of Earth," Planetary and Space Science 74, 202-207 (Dec 2012).

[22] Hoeijmakers, H. J., Arts, M. L. J., Snik, F., Keller, C. U., Stam, D. M., and Kuiper, J. M., "Design tradeoff and proof of concept for LOUPE, the Lunar Observatory for Unresolved Polarimetry of Earth," Optics Express 24, 21435 (Sep 2016).

[23] Sparks, W., Hough, J. H., Germer, T. A., Robb, F., and Kolokolova, L., "Remote sensing of chiral signatures on Mars," Planetary and Space Science 72, 111-115 (Nov 2012). 
[24] Ruane, G., Riggs, A., Mazoyer, J., Por, E. H., N’Diaye, M., Huby, E., Baudoz, P., Galicher, R., Douglas, E., Knight, J., Carlomagno, B., Fogarty, K., Pueyo, L., Zimmerman, N., Absil, O., Beaulieu, M., Cady, E., Carlotti, A., Doelman, D., Guyon, O., Haffert, S., Jewell, J., Jovanovic, N., Keller, C., Kenworthy, M. A., Kuhn, J., Miller, K., Sirbu, D., Snik, F., Wallace, J. K., Wilby, M., and Ygouf, M., "Review of high-contrast imaging systems for current and future ground- and space-based telescopes I: coronagraph design methods and optical performance metrics," in [Space Telescopes and Instrumentation 2018: Optical, Infrared, and Millimeter Wave], Society of Photo-Optical Instrumentation Engineers (SPIE) Conference Series 10698, 106982S (Aug 2018).

[25] Jovanovic, N., Absil, O., Baudoz, P., Beaulieu, M., Bottom, M., Cady, E., Carlomagno, B., Carlotti, A., Doelman, D., Fogarty, K., Galicher, R., Guyon, O., Haffert, S., Huby, E., Jewell, J., Keller, C., Kenworthy, M. A., Knight, J., Kühn, J., Miller, K., Mazoyer, J., N’Diaye, M., Por, E., Pueyo, L., Riggs, A. J. E., Ruane, G., Sirbu, D., Snik, F., Wallace, J. K., Wilby, M., and Ygouf, M., "Review of high-contrast imaging systems for current and future ground-based and space-based telescopes: Part II. Common path wavefront sensing/control and coherent differential imaging," in [Adaptive Optics Systems VI], Society of Photo-Optical Instrumentation Engineers (SPIE) Conference Series 10703, 107031U (Jul 2018).

[26] Snik, F., Absil, O., Baudoz, P., Beaulieu, M., Bendek, E., Cady, E., Carlomagno, B., Carlotti, A., Cvetojevic, N., Doelman, D., Fogarty, K., Galicher, R., Guyon, O., Haffert, S., Huby, E., Jewell, J., Jovanovic, N., Keller, C., Kenworthy, M. A., Knight, J., Kuhn, J., Mazoyer, J., Miller, K., N’Diaye, M., Norris, B., Por, E., Pueyo, L., Riggs, A. J. E., Ruane, G., Sirbu, D., Wallace, J. K., Wilby, M., and Ygouf, M., "Review of high-contrast imaging systems for current and future ground-based and space-based telescopes III: technology opportunities and pathways," in [Advances in Optical and Mechanical Technologies for Telescopes and Instrumentation III], Society of Photo-Optical Instrumentation Engineers (SPIE) Conference Series 10706, 107062L (Jul 2018).

[27] Snik, F. and Keller, C. U., "Astronomical Polarimetry: Polarized Views of Stars and Planets," in [Planets, Stars and Stellar Systems, by Oswalt, Terry D.; Bond, Howard E., ISBN 978-94-007-5617-5. Springer Science+Business Media Dordrecht, 2013, p. 175], Oswalt, T. D. and Bond, H. E., eds., 175, Springer (2013).

[28] Snik, F., Craven-Jones, J., Escuti, M., Fineschi, S., Harrington, D., De Martino, A., Mawet, D., Riedi, J., and Tyo, J. S., "An overview of polarimetric sensing techniques and technology with applications to different research fields," in [Polarization: Measurement, Analysis, and Remote Sensing XI], Society of Photo-Optical Instrumentation Engineers (SPIE) Conference Series 9099, 90990B (May 2014).

[29] Bagnulo, S., Landolfi, M., Landstreet, J. D., Land i Degl'Innocenti, E., Fossati, L., and Sterzik, M., "Stellar Spectropolarimetry with Retarder Waveplate and Beam Splitter Devices," PASP 121, 993 (Sep 2009 ).

[30] Parejo, P. G. and Álvarez Herrero, A., "Liquid crystals for space instrumentation: optical properties of liquid crystal mixtures for polarimeters," Opt. Mater. Express 9, 2681-2698 (Jun 2019).

[31] Kudenov, M. W., "Channeled polarimetry for snapshot measurements; ISBN 9781439830406," in [Polarized Light, 3rd Edition], Goldstein, D. H., ed., 808, CRC Press (2010).

[32] Oka, K. and Kato, T., "Spectroscopic polarimetry with a channeled spectrum," Opt. Lett. 24, 1475-1477 (Nov 1999).

[33] Snik, F., Karalidi, T., and Keller, C. U., "Spectral modulation for full linear polarimetry," Appl. Opt. 48, 1337-1346 (Mar 2009).

[34] Sparks, W., Germer, T. A., MacKenty, J. W., and Snik, F., "Compact and robust method for full Stokes spectropolarimetry," Applied Optics 51, 5495 (Aug 2012).

[35] Sparks, W. B., Germer, T. A., and Sparks, R. M., "Classical Polarimetry with a Twist: A Compact, Geometric Approach," Publications of the Astronomical Society of the Pacific 131, 075002 (Jul 2019 ).

[36] Miskiewicz, M. N. and Escuti, M. J., "Direct-writing of complex liquid crystal patterns," Opt. Express 22, 12691-12706 (May 2014).

[37] Komanduri, R. K., Lawler, K. F., and Escuti, M. J., "Multi-twist retarders: broadband retardation control using self-aligning reactive liquid crystal layers," Opt. Express 21, 404-420 (Jan 2013).

[38] Oh, C. and Escuti, M. J., "Achromatic diffraction from polarization gratings with high efficiency," Opt. Lett. 33, 2287-2289 (Oct 2008). 
[39] Packham, C., Escuti, M., Ginn, J., Oh, C., Quijano, I., and Boreman, G., "Polarization Gratings: A Novel Polarimetric Component for Astronomical Instruments," Publications of the Astronomical Society of the Pacific 122, 1471 (Dec 2010).

[40] Escuti, M. J., Kim, J., and Kudenov, M. W., "Controlling light with geometric-phase holograms," Opt. Photon. News 27, 22-29 (Feb 2016).

[41] Snik, F., Rietjens, J. H. H., van Harten, G., Stam, D. M., Keller, C. U., Smit, J. M., Laan, E. C., Verlaan, A. L., Ter Horst, R., Navarro, R., Wielinga, K., Moon, S. G., and Voors, R., "SPEX: the spectropolarimeter for planetary exploration," in [Space Telescopes and Instrumentation 2010: Optical, Infrared, and Millimeter Wave], Society of Photo-Optical Instrumentation Engineers (SPIE) Conference Series 7731, 77311B (Jul 2010).

[42] van Harten, G., de Boer, J., Rietjens, J. H. H., Di Noia, A., Snik, F., Volten, H., Smit, J. M., Hasekamp, O. P., Henzing, J. S., and Keller, C. U., "Atmospheric aerosol characterization with a ground-based SPEX spectropolarimetric instrument," Atmospheric Measurement Techniques 7, 4341-4351 (Dec 2014).

[43] Rietjens, J. H. H., Chanumolu, A., Campo, J., Nalla, R., Fernandez, C. V., Dingjan, J., Smit, M., Snik, F., van Amerongen, A., and Hasekamp, O. P., "Expected performance and error analysis for SPEXone, a multi-angle channeled spectropolarimeter for the NASA PACE mission," in [Polarization Science and Remote Sensing IX], Society of Photo-Optical Instrumentation Engineers (SPIE) Conference Series 11132, 11132-8 (Aug 2019).

[44] Kemp, J. C., Henson, G. D., Steiner, C. T., and Powell, E. R., "The optical polarization of the Sun measured at a sensitivity of parts in ten million," Nature 326, 270-273 (Mar 1987). 